\title{
A New Treatment Option for Intermediate- Stage Hepatocellular Carcinoma with High Tumor Burden: Initial Lenvatinib Therapy with Subsequent Selective TACE
}

Masatoshi Kudo

Department of Gastroenterology and Hepatology, Kindai University Faculty of Medicine, Osaka-Sayama, Japan

Prof. M. Kudo

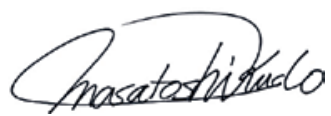

Editor Liver Cancer

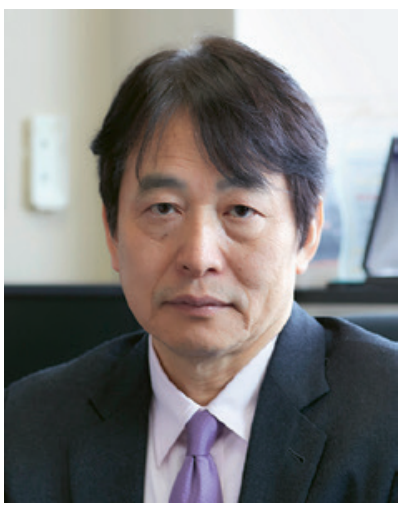

\section{Introduction}

Transarterial chemoembolization (TACE) is the only guideline-recommended global standard of care for intermediate-stage hepatocellular carcinoma (HCC). However, TACE is not beneficial for three subgroups of patients with the following characteristics: (1) conditions that easily become refractory to TACE, (2) conditions in which TACE causes deterioration of hepatic functional reserve to Child-Pugh class B, and (3) conditions that are unlikely to benefit from TACE. In the first subgroup, which includes HCCs beyond the up-to-seven criteria, the response to TACE is typically poor, and hepatic functional reserve is further impaired. This may hinder the switch to molecular-targeted therapy, resulting in a worse prognosis and shorter overall survival. Recent evidence indicates that initial lenvatinib therapy followed by TACE markedly improves overall survival in this TACE-unsuitable subpopulation. Thus, lenvatinib-TACE sequential therapy (LEN-TACE sequential therapy) may become the standard of care for patients who do not benefit from TACE. 

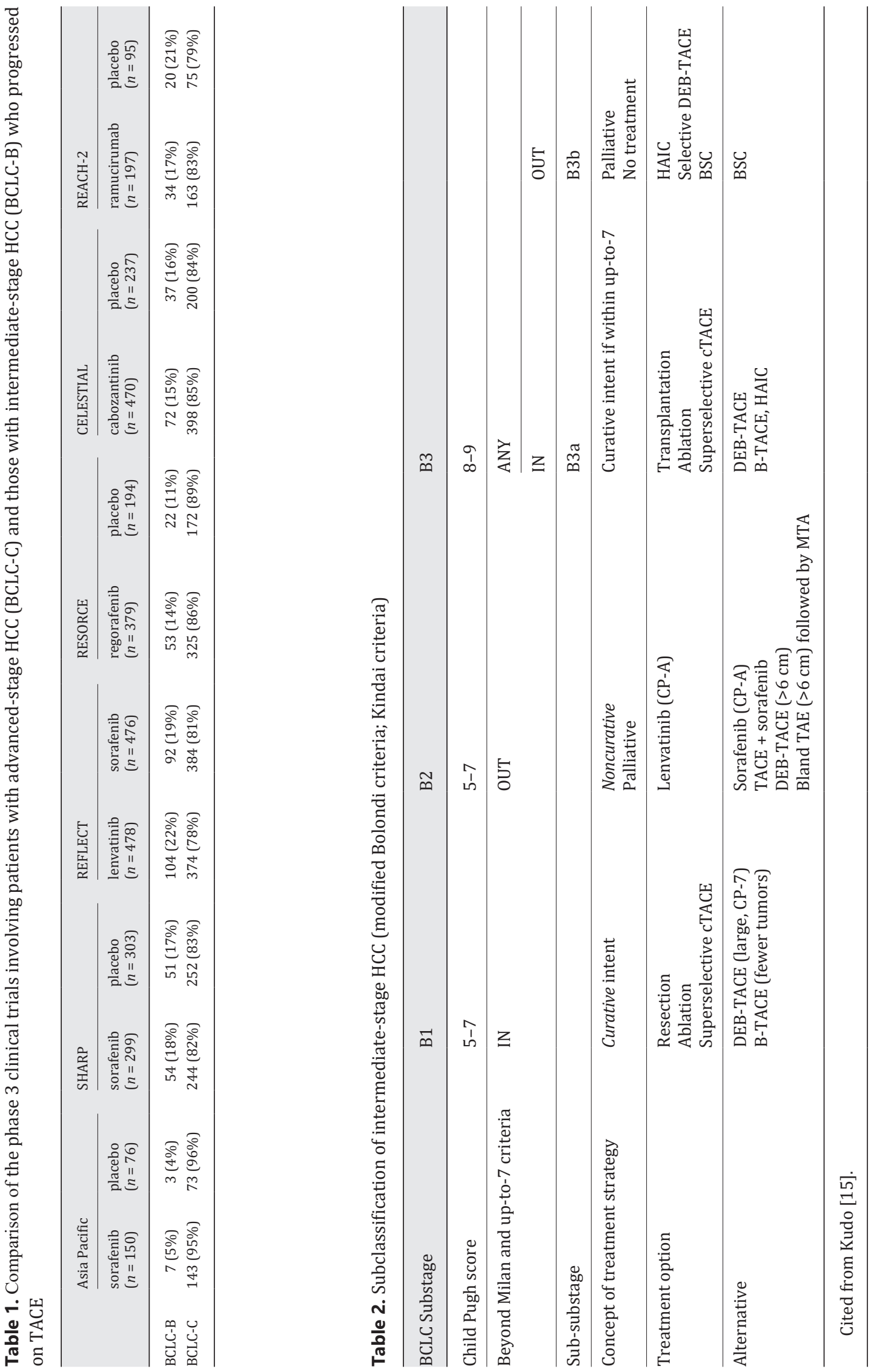


\section{Liver Cancer}

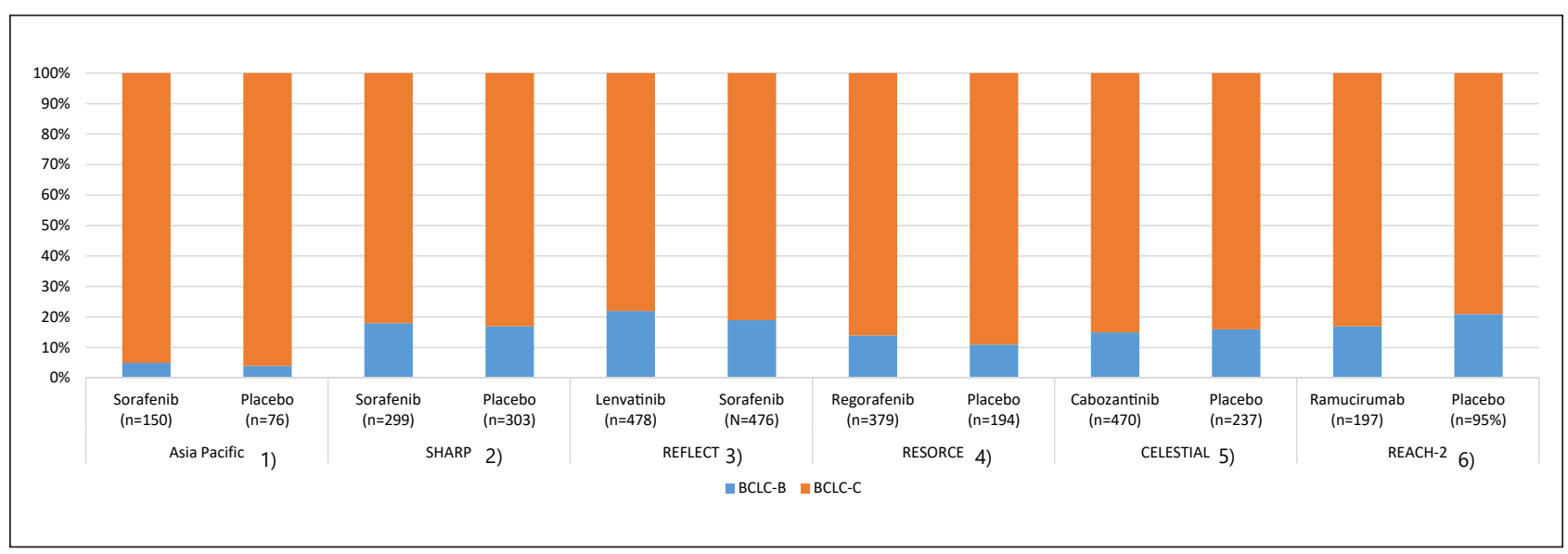

Fig. 1. Ratio of intermediate-stage HCC among phase 3 positive trials in unresectable HCC. HCC, hepatocellular carcinoma.

The recent approval in 2019 of ramucirumab and cabozantinib for the treatment of HCC increased the number of molecular-targeted agents (MTAs) available in clinical practice to five: lenvatinib and sorafenib as first-line agents, and regorafenib, cabozantinib, and ramucirumab as second-line agents [1-6]. The survival benefits of the five agents were demonstrated in phase 3 clinical trials involving patients with advanced-stage HCC (Barcelona Clinic Liver Cancer [BCLC]-C) and those with intermediate-stage HCC (BCLC-B) who progressed on TACE. Approximately $20 \%$ of the patients included in these clinical trials (except the AsiaPacific trial on sorafenib) had intermediate-stage HCC (Table 1; Fig. 1) [1-6]. Most of the patients with intermediate-stage HCC enrolled in these trials were refractory to TACE after multiple sessions.

An important issue that needs to be addressed is the establishment of treatment strategies for the largest proportion of patients; namely, those with intermediate-stage HCC (especially those who will not benefit from TACE in the multi-MTA era). Once liver function has deteriorated to Child-Pugh class B it is often too late to start molecular-targeted therapy. To improve the overall survival of HCC patients in the current multi-MTA era, molecular-targeted therapy should be introduced under the following conditions: intermediate-stage HCC that is refractory to TACE or in which TACE is unlikely to be effective and liver function is still well preserved. In these cases, different agents should be used sequentially to extend patient survival. Lenvatinib therapy, which is associated with a high response rate, followed by selective TACE may be an effective treatment strategy. This editorial explains the concept of TACE unsuitability and outlines new treatment strategies for TACE-unsuitable intermediatestage HCC in the era of multiple MTAs.

\section{Heterogeneity of Intermediate-Stage HCC and Treatment Strategies}

Intermediate-stage HCC is extremely heterogeneous in terms of (1) hepatic functional reserve, (2) tumor size, and (3) tumor number [7]. More precisely, hepatic functional reserve varies widely in Child-Pugh class: from class A (score 5) to class B (score 9), tumor size: from $\geq 5 \mathrm{~cm}$ to huge $(>10 \mathrm{~cm})$, and number of nodules: from 4 to $>10$. Despite such extreme heterogeneity, TACE is the only standard of care recommended by guidelines worldwide [8-11]. Because there are currently five available agents for the treatment of HCC, a change in the guidelines may be necessary. Dr. Bruix, who originally proposed the BCLC staging system, 
Table 3. Liver function at TACE refractoriness

\begin{tabular}{llrll}
\hline & Year & $n$ & & \multicolumn{2}{l}{ Child-Pugh class } \\
\cline { 3 - 4 } & & & $\mathrm{A}$ & $\mathrm{B} / \mathrm{C}$ \\
\hline Ogasawara [23] & 2014 & 122 & $75 \%$ & $25 \%$ \\
Arizumi [24] & 2014 & 56 & $80 \%$ & $20 \%$ \\
OPTIMIS [25] & 2019 & 636 & $64 \%$ & $26 \%$ \\
\hline
\end{tabular}

At the time of TACE refractoriness, liver function has already decreased, and there are many patients who are not good candidates for systemic therapy. TACE refractoriness is too late when considering systemic therapy.

stated in a review article published in Gastroenterology that TACE is not indicated for all cases of intermediate-stage HCC, and should only be used in cases of solitary or limited multinodular HCC with preserved liver function [12]. Therefore, the development of treatment strategies for multinodular disease in both lobes and large tumors constitutes an unmet need.

Various systems for subclassification of intermediate-stage HCC and treatment strategies for each substage have been proposed [13-16] (Table 2). For subclassification of intermediate-stage HCC, the up-to-seven criteria originally developed as criteria for transplantation [17] are widely used globally to describe the level of tumor burden.

\section{Criteria for TACE Refractoriness and the Timing of a Therapy Switch to Molecular- Targeted Therapy}

In 2011, the Japan Society of Hepatology (JSH) established the criteria for refractoriness to TACE for the first time in the world, followed by revision in $2014[18,19]$. The current criteria for TACE refractoriness published in different countries include (1) the JSH criteria [18, 19], (2) criteria proposed by Raoul et al. [20], (3) Taiwanese criteria [21], and (4) criteria proposed by the International Expert Panel (EPOI HCC) [22]. The JSH defines refractoriness to TACE as failure to control target lesions or the appearance of new lesions even after two or more consecutive TACE sessions. Raoul et al. [20] recommend a treatment switch after two consecutive insufficient responses to TACE. The EPOI HCC and Taiwanese criteria define TACE refractoriness as that occurring within 6 months after $\geq 2$ or $\geq 3$ on-demand TACE sessions, and recommend an immediate switch to molecular-targeted therapy when these criteria are met. In Taiwan, these criteria must be met to be reimbursed for the MTA sorafenib. The JSH guidelines might have changed the Taiwanese reimbursement policy. Based on the JSH guidelines recommending an immediate switch to sorafenib at the point of TACE refractoriness, sorafenib (which was previously limited to treatment of advanced HCC in Taiwan) became a reimbursable drug for the treatment of intermediate-stage HCCs that meet the EPOI HCC criteria for TACE refractoriness.

Two retrospective studies demonstrated better maintenance of hepatic functional reserve and a longer time-to-disease progression in the sorafenib switch group than in the repeat TACE group. Patients who met TACE refractoriness criteria and switched to sorafenib rather than continuing ineffective TACE therapy showed prolonged overall survival $[23,24]$. A global noninterventional prospective study termed OPTIMIS, which was conducted to assess the outcomes of TACE-refractory patients, also showed that the group that switched to sorafenib immediately after becoming refractory to TACE had a better prognosis than the group that did not switch to sorafenib even after the point of TACE refractoriness [25]. 


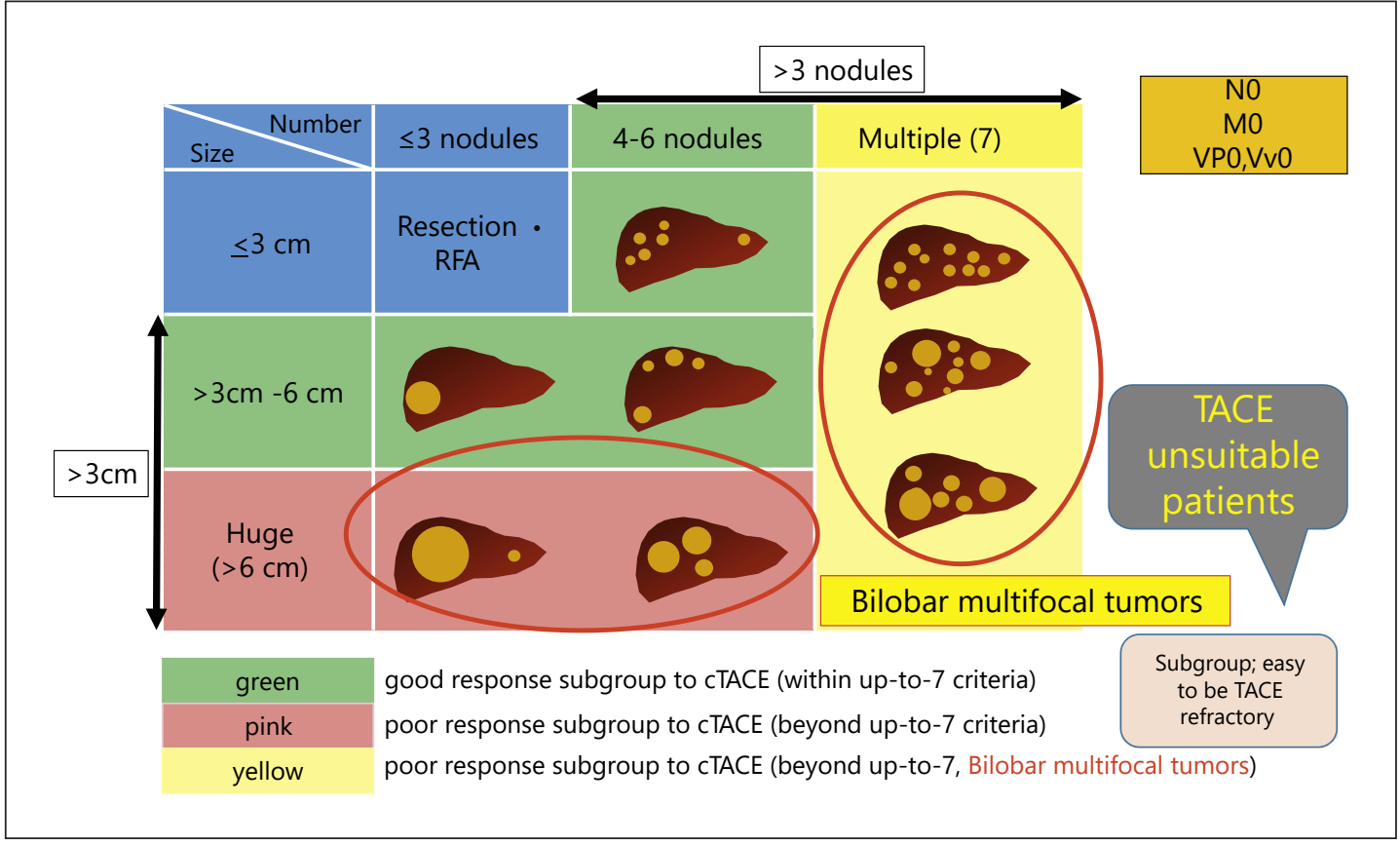

Fig. 2. Heterogeneity and treatment strategy of intermediate-stage HCC.

\section{Should a Switch from TACE to Molecular-Targeted Therapy Be Delayed until the Point of TACE Refractoriness?}

An immediate switch to an MTA after the point of TACE refractoriness is gaining consensus worldwide. However, closer review of the three major studies mentioned earlier indicates that liver function had already dropped to Child-Pugh class B or C in approximately $20-26 \%$ of patients at the time of TACE refractoriness [23-25] (Table 3). Therefore, switching to molecular-targeted therapy is difficult in a considerable proportion of patients who continue TACE until becoming refractory to it, and this change in treatment should be made at an earlier time point than that recommended by existing guidelines in the current multi-MTA era.

\section{The Concept of "TACE Unsuitability" in Intermediate-Stage HCC}

The concept of TACE unsuitability was recently proposed [15]. TACE unsuitability refers to the following three major conditions: (1) conditions that easily become refractory to TACE, (2) conditions in which TACE is associated with deterioration of hepatic functional reserve to Child-Pugh class B, and (3) conditions that are unlikely to respond to TACE.

First, HCCs with tumor burden (tumor size and number) beyond the up-to-seven criteria easily become refractory to TACE [26-29] (Fig. 2). Hepatic functional reserve tends to deteriorate after TACE in patients with HCC beyond the up-to-seven criteria and those with albumin-bilirubin (ALBI) grade $2[27,30]$. A decrease in the ALBI score is positively associated with the number of TACE sessions [31]. Therefore, even one TACE session may worsen hepatic functional reserve in ALBI grade 2 patients, and this could result in a missed chance for the introduction of molecular-targeted therapy. In addition, TACE may not be effective in the following tumor phenotypes: tumors without a clear boundary (such as a single nodular 


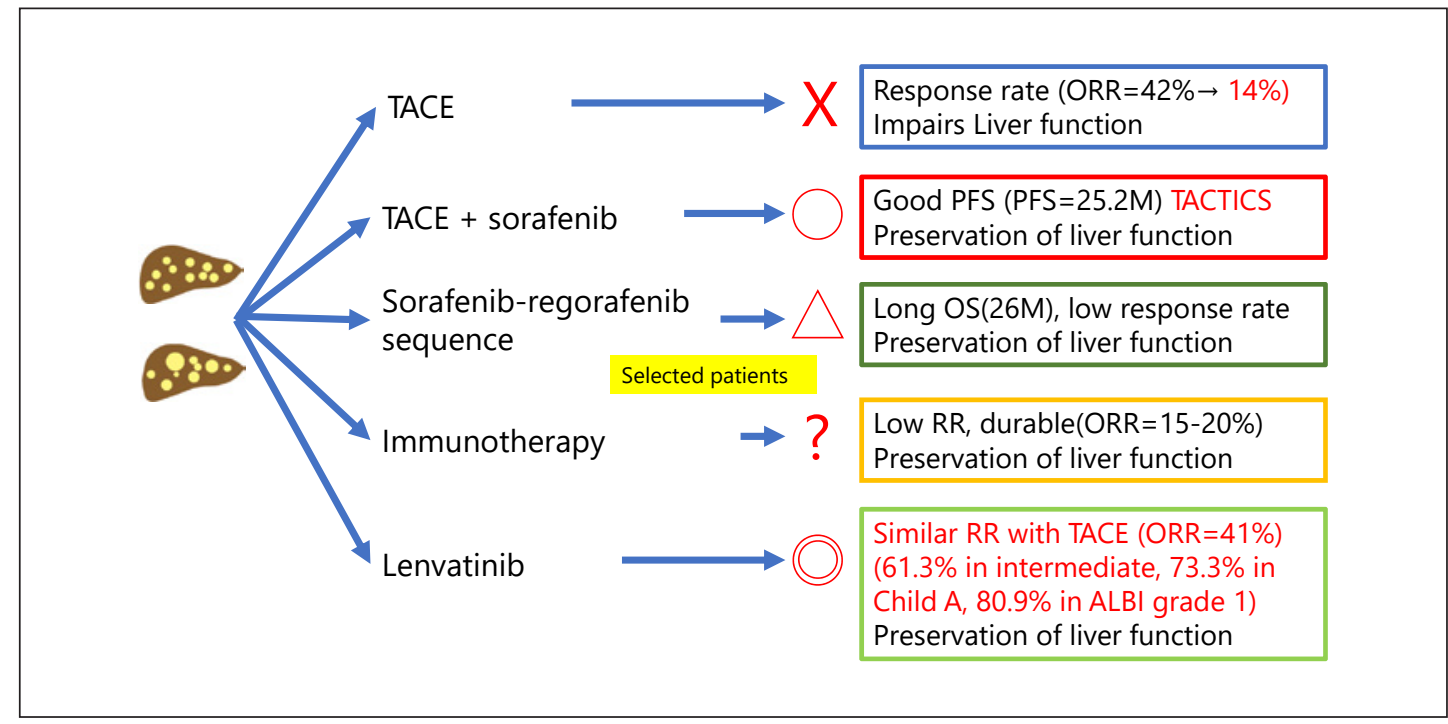

Fig. 3. Treatment strategy of bilobar multifocal intermediate-stage HCC (TACE-unsuitable HCC).

Table 4. TACE-unsuitable patient subpopulation

A. Conditions that easily become refractory to TACE:

- Beyond up-to-seven criteria

B. Conditions in which TACE causes deterioration of liver function to Child-Pugh class B:

- Beyond up-to-seven criteria

- $\quad$ ALBI grade 2

C. Conditions that are unlikely to respond to TACE (TACE-resistant tumor):

- Simple nodular type tumor with extranodular growth

- Confluent multinodular type tumor

- Massive type tumor

- Poorly differentiated HCC

- Intrahepatic multifocal metastasis

- Sarcomatous change caused by TACE

tumor type with extranodular growth), confluent multinodular type tumors, and massive type tumors [32] (which have an increased risk of microvascular invasion) as well as poorly differentiated HCC [33]. These three conditions are unlikely to benefit from TACE, and repeated TACE in patients who become refractory can deteriorate liver function to ChildPugh class B (Table 4). In these cases, molecular-targeted therapy cannot be introduced, which may shorten overall survival.

\section{Outcome of Initial Lenvatinib Therapy with Subsequent TACE in a Subpopulation of Primary TACE-Unsuitable Patients}

Previously, the concept was proposed that lenvatinib as an initial treatment for TACEunsuitable intermediate-stage HCC patients (e.g., bilobar multifocal HCC) may improve prognosis [15] (Fig. 3, 4). A recent proof-of-concept study showed that lenvatinib as the initial treatment followed by subsequent selective TACE resulted in favorable outcomes in patients 


\section{Liver Cancer}

\begin{tabular}{l|l}
\hline \multicolumn{2}{l}{ Liver Cancer 2019;8:299-311 } \\
\hline DOI: 10.1159/000502905 & $\begin{array}{l}\text { @ 2019 S. Karger AG, Basel } \\
\text { www.karger.com/lic }\end{array}$ \\
\hline
\end{tabular}

Kudo: New Treatment Strategy for TACE-Unsuitable Intermediate-Stage HCC

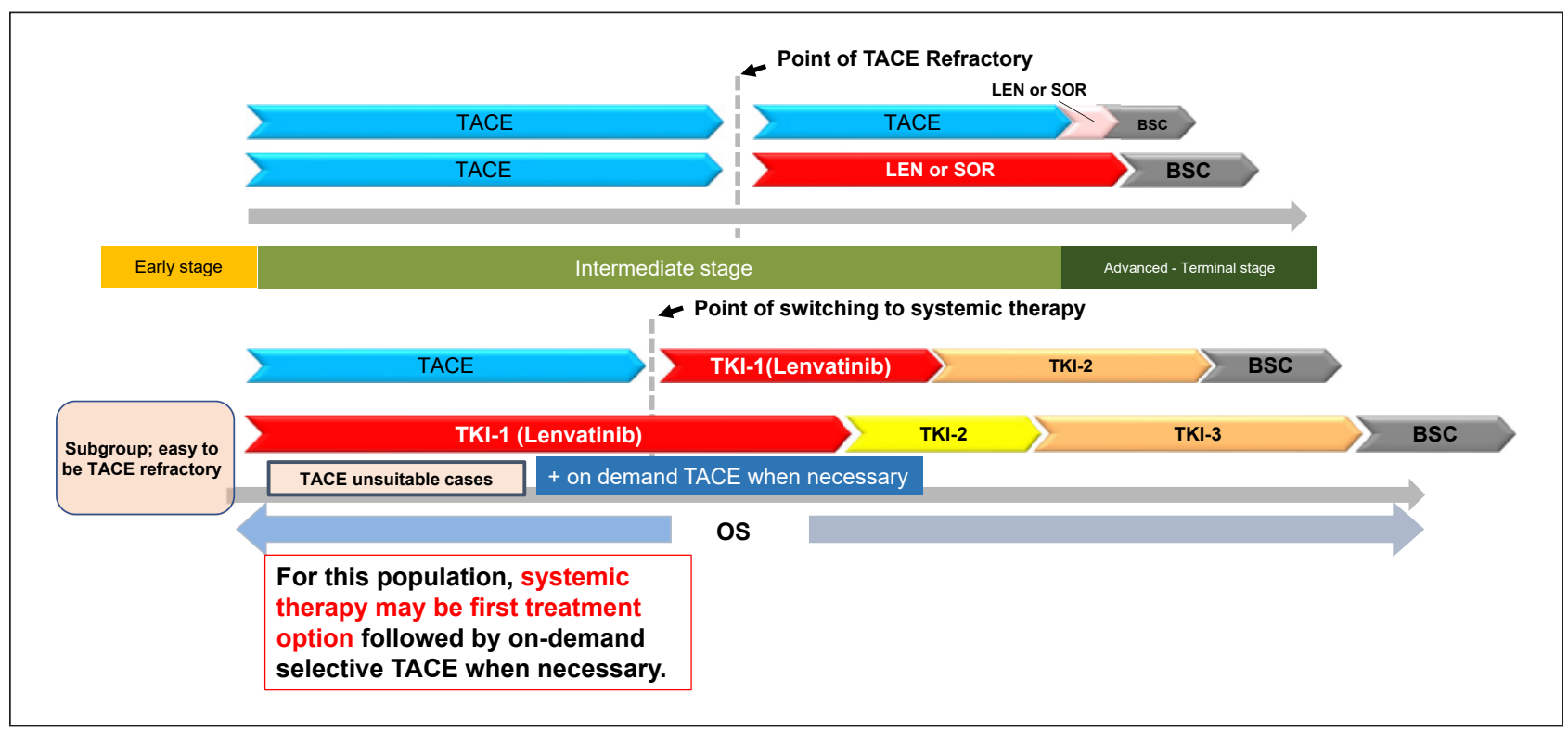

Fig. 4. Treatment strategy of intermediate-stage HCC.

Table 5. Change of ALBI score in proof-of-concept study

\begin{tabular}{llllll}
\hline & Baseline & 1 month & 2 months & 3 months & EOT \\
\hline Lenvatinib & -2.61 & -2.45 & $-2.40^{*}$ & $-2.49^{* *}$ & -2.61 \\
TACE & -2.66 & $-2.25^{\#}$ & $-2.24^{\#, *}$ & $-2.15^{\#, * *}$ & $-2.09^{\#}$ \\
\hline
\end{tabular}

Cited and modified from Kudo et al. [34]. ALBI score, albumin-bilirubin score; EOT, end of treatment; TACE, transcatheter arterial chemoembolization. ${ }^{*} p<0.05$, lenvatinib vs. TACE; ${ }^{* *} p<0.01$, lenvatinib vs. TACE; ${ }^{\#} p<0.01$, vs. TACE at baseline.

with HCC beyond the up-to-seven criteria (a TACE-unsuitable patient subpopulation) [34]. This clinical study compared the treatment outcomes of intermediate-stage HCC beyond the up-to-seven criteria and Child-Pugh class A liver function between 37 TACE-naïve patients who received lenvatinib as the initial treatment (lenvatinib group) and 139 consecutive patients with HCC beyond the up-to-seven criteria who underwent TACE as the initial treatment (TACE group) during the same period. Propensity score matching was used to adjust the baseline characteristics. Efficacy was compared between 30 patients in the lenvatinib group (excluding seven patients with a follow-up period $\leq 6$ months) and 60 propensity score-matched patients in the TACE group. The results showed that TACE irreversibly decreased hepatic functional reserve as determined by the ALBI score, whereas liver function was maintained in the lenvatinib group at the end of treatment (Table 5). Progression-free survival was significantly longer in the lenvatinib group than in the TACE group (16 vs. 3 months, respectively; hazard ratio, $0.19 ; 95 \%$ confidence interval, $0.10-0.35 ; p<0.001$; Table 6). Overall survival was markedly longer in the lenvatinib group than in the TACE group (37.9 vs. 21.3 months, respectively; hazard ratio, 0.48 ; 95\% confidence interval, $0.16-0.79 ; p<0.01$; Table 6).

Approximately $70 \%$ of patients underwent TACE after initial lenvatinib therapy in the lenvatinib group. Four patients in the lenvatinib group achieved cancer-free and drug-free 
Table 6. OS and PFS of initial lenvatinib treatment with subsequent selective TACE in proof-of-concept study

\begin{tabular}{llcr}
\hline & $\begin{array}{l}\text { Lenvatinib (followed } \\
\text { by selective TACE) }\end{array}$ & TACE & $\begin{array}{l}\text { HR } \\
p \text { value }\end{array}$ \\
\hline Median OS, months & $37.9(23.1-\mathrm{NR})$ & $21.3(15.7-28.4)$ & $\begin{array}{c}0.48(0.16-0.79) \\
<0.01\end{array}$ \\
\hline Median PFS, months & $16.0(10.9-16.6)$ & $3.0(2.1-4.3)$ & $\begin{array}{r}0.19(0.10-0.35) \\
<0.001\end{array}$
\end{tabular}

Values in parentheses are 95\% CI. Cited and modified from Kudo et al. [34]. TACE, transcatheter arterial chemoembolization; OS, overall survival; PFS, progression-free survival; NR, not reached.

Table 7. Objective response rate of lenvatinib and TACE in proof-of-concept study

\begin{tabular}{lllc}
\hline & $\begin{array}{l}\text { Lenvatinib } \\
(n=30)\end{array}$ & $\begin{array}{l}\text { TACE } \\
(n=60)\end{array}$ & $\begin{array}{l}p \text { value } \\
\text { Odds ratio }(95 \% \mathrm{CI})\end{array}$ \\
\hline ORR & $22(73.3 \%)$ & $20(33.3 \%)$ & $<0.001$ \\
CBR (CR + PR + SD $\geq 24$ weeks) & $29(96.7 \%)$ & $22(36.7 \%)$ & $<.39(1.90-16.67)$ \\
DCR & $30(100.0 \%)$ & $33(55.0 \%)$ & $48.1(7.01-2,073.85)$ \\
CR & 2 & 4 & \\
PR & 20 & 16 & \\
SD & 8 & 12 & \\
Durable SD (SD $\geq 24$ weeks) & 7 & 2 & \\
PD & 0 & 26 & \\
NE & 0 & 2 & \\
\hline
\end{tabular}

ORR, objective response rate; CBR, clinical benefit rate; $C R$, complete response; PR, partial response; $\mathrm{SD}$, stable disease; PD, progressive disease; NE, not evaluable. Cited from Kudo et al. [34].

status after a complete response, of which one achieved drug-free status with lenvatinib only. This extremely favorable outcome of initial lenvatinib therapy with subsequent selective TACE occurred in patients with intermediate-stage HCC beyond the up-to-seven criteria, which is extremely difficult to manage using TACE alone. This underscores the important role of lenvatinib, which is associated with high response rates in patients with intermediatestage HCC beyond the up-to-seven criteria, as the first choice of treatment. The advantage of lenvatinib followed by selective TACE over TACE alone with respect to overall survival was attributed to the high response rates and preservation of liver function associated with lenvatinib treatment. The response rate of lenvatinib was $40.6 \%$ in the REFLECT trial [3] and $61.3 \%$ in a Japanese population with intermediate-stage HCC in the REFLECT study [35], and was extremely high (73.3\%) in the proof-of-concept study [34] (Table 7). The high response rate may be related to the administration of lenvatinib at a full dose for a long period of time, which was possible because a large proportion of TACE-naïve patients had well-preserved hepatic functional reserve (mainly within ALBI grade 1); the low rates of dose reduction, interruption, and discontinuation because of adverse events were low [36]. The benefits of lenvatinib pretreatment are as follows: (1) tumor shrinkage and/or necrosis due to high response rate of lenvatinib [3]; (2) it enables curative TACE and the preservation of hepatic functional reserve; (3) achievement of drug-free complete response attributed to an opti- 


\section{Liver Cancer}

Fig. 5. Effect on tumor vasculature by VEGF inhibition. Adapted from Jain [38].

\begin{tabular}{l|l}
\hline \multicolumn{2}{l}{ Liver Cancer 2019;8:299-311 } \\
\hline DOI: 10.1159/000502905 & $\begin{array}{l}\text { @ 2019 S. Karger AG, Basel } \\
\text { www.karger.com/lic }\end{array}$ \\
\hline
\end{tabular}

Kudo: New Treatment Strategy for TACE-Unsuitable Intermediate-Stage HCC

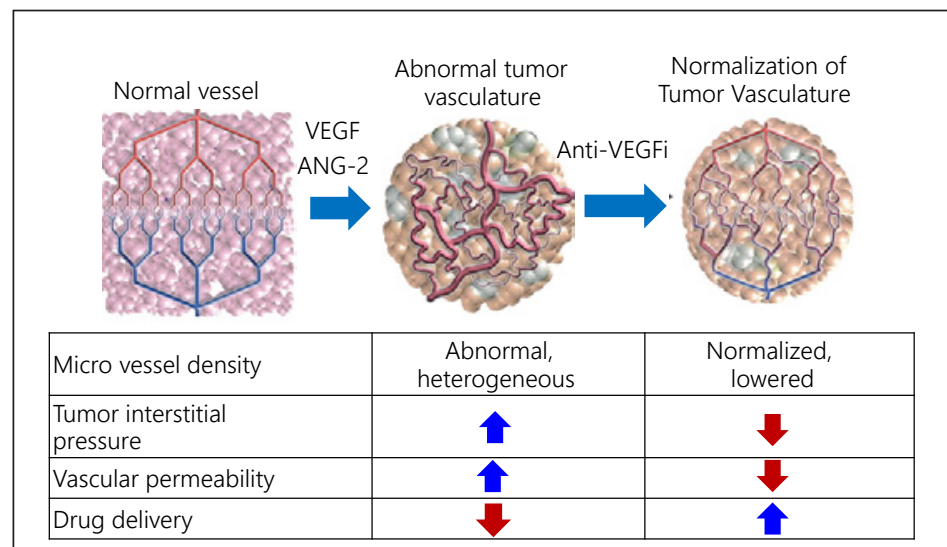

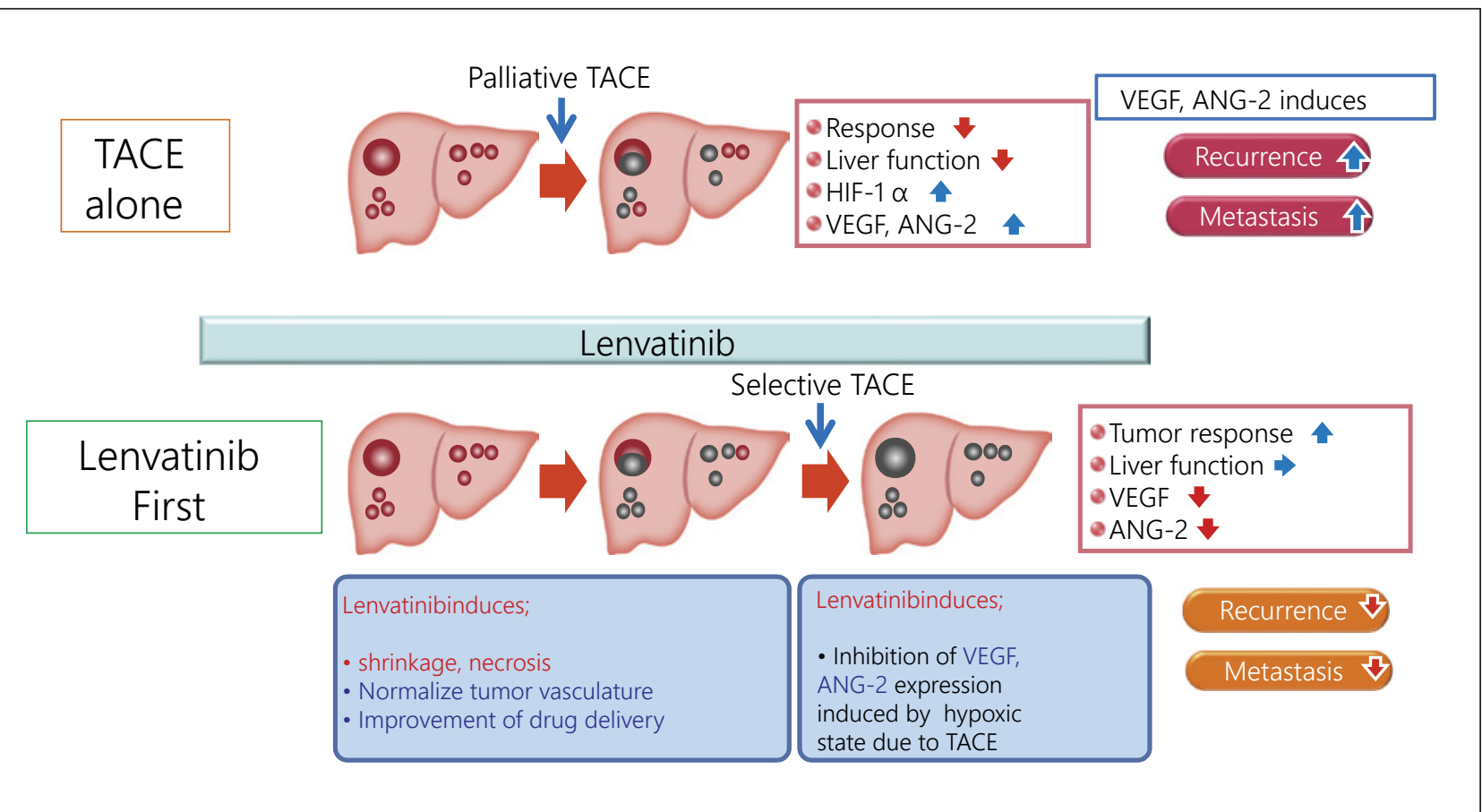

Fig. 6. Synergistic effect by initial lenvatinib treatment with subsequent selective TACE. Initial lenvatinib treatment maximizes the effect of TACE.

mized embolization effect because targeted agents improve drug delivery [37]; vascular normalization [38] by lenvatinib and the consequent reduction in vascular permeability and tumor interstitial pressure improves the distribution of lipiodol-containing anticancer drugs within the tumor [38]; and (4) prevention of recurrence and metastasis [39, 40] through inhibition of hypoxia-inducible factors such as VEGF following TACE [37, 38] (Fig. 5, 6). These features suggest the efficacy of LEN-TACE sequential therapy in patients with intermediatestage HCC with a high tumor burden, such as HCCs beyond the up-to-seven criteria (Fig. 6, 7). No disadvantages of lenvatinib pretreatment before TACE have been identified, at least in high tumor burden HCCs, indicating a possible paradigm shift in HCC treatment (Fig. 8). LEN-TACE sequential therapy may become the standard of care for patients who do not benefit from TACE (TACE-unsuitable patients) (Fig. 7, 8). 

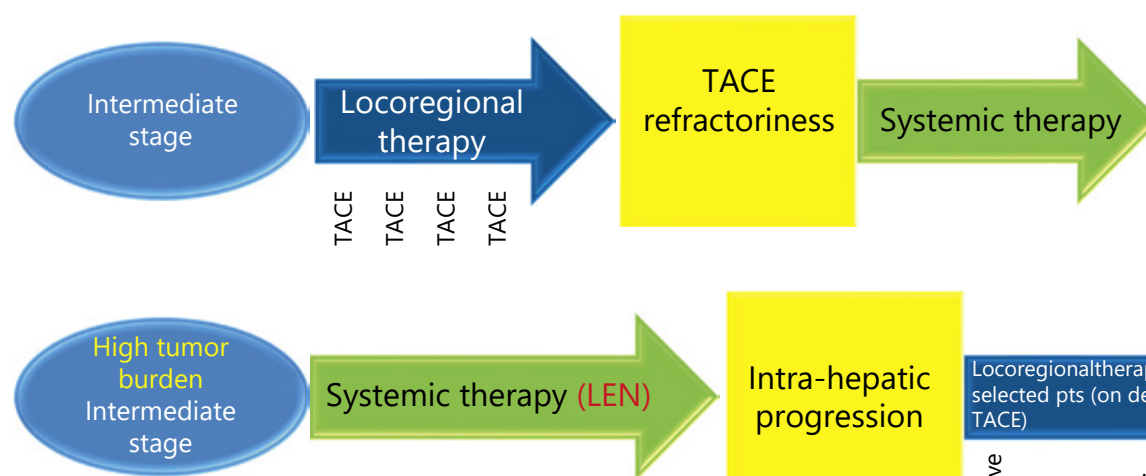

Systemic therapy (LEN)
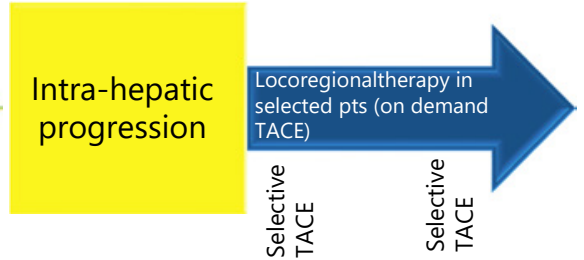

Fig. 7. Changing concept in intermediate-stage HCC with high tumor burden.

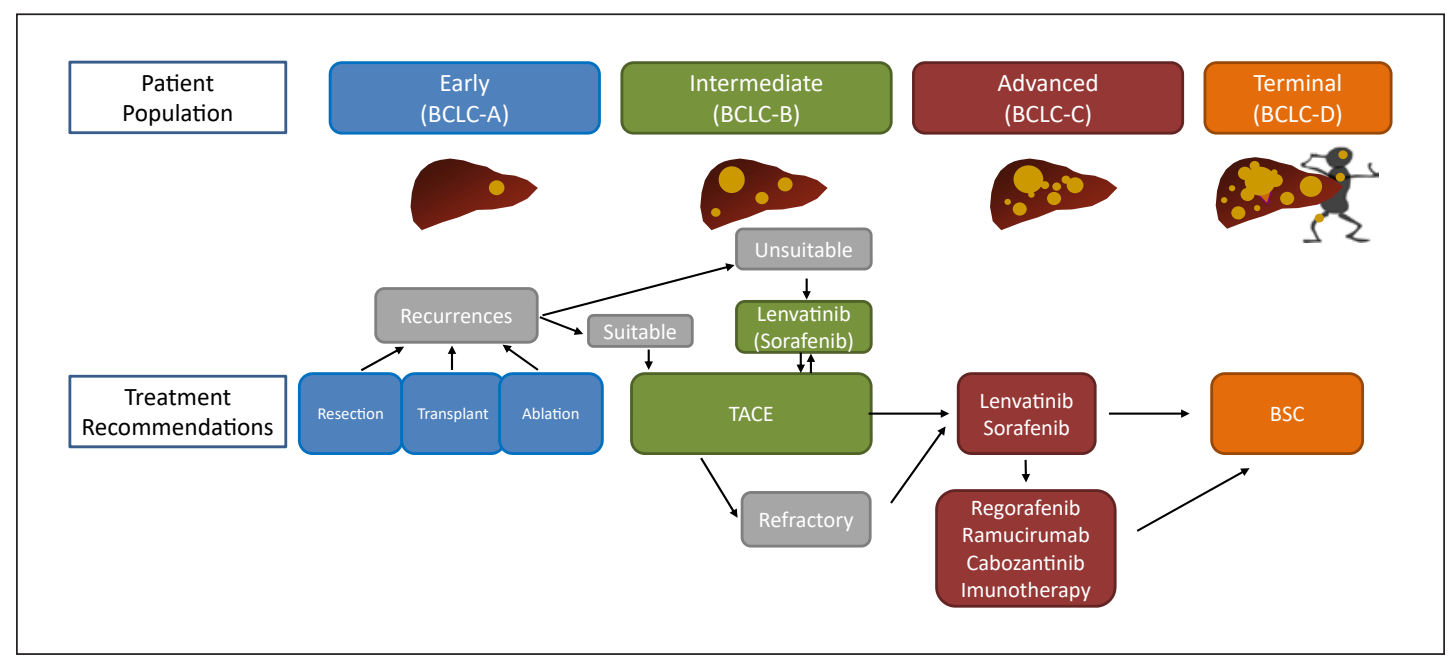

Fig. 8. New paradigm of treatment strategy in HCC.

LEN-TACE sequential therapy is expected to elicit a high response rate without affecting hepatic functional reserve in patients with bilobar multinodular disease. In large tumors $(\geq 5$ $\mathrm{cm})$, TACE often needs to be split into several lipiodol-TACE sessions because the lipiodol volume of a single TACE session is not sufficient to fill the entire tumor. This increases the risk of recurrence and metastasis due to the release of hypoxia-inducible factors from the residual tumor between TACE sessions, leading to increased VEGF, FGF, and angiopoietin-2. Drugeluting bead-TACE, which is sometimes used for the treatment of large tumors or bilobar multifocal tumors, is associated with the risk of residual cancer cells in the tumor periphery. This can cause phenotypic changes to sarcomatous or cholangiocarcinoma-like mixed phenotypes [41, 42], which are associated with recurrence, metastasis, and invasion upon release of VEGF, FGF, or angiopoietin-2 [43, 44]. The effects of LEN-TACE on normalizing tumor neovasculature, preventing post-TACE increases in serum VEGF level, and reducing residual tumor volume suggest that this strategy will yield excellent therapeutic effects and preserve liver function in patients with bilobar multinodular HCCs and huge HCCs (Fig. 5, 6). 
Kudo: New Treatment Strategy for TACE-Unsuitable Intermediate-Stage HCC

Because of its efficacy for the treatment of TACE-unsuitable patients with HCC beyond the up-to-seven criteria who can become refractory to TACE, lenvatinib may become the first choice of treatment in the following patient populations: high tumor burden intermediatestage HCC, conditions refractory to TACE, and conditions with ALBI grade 2 hepatic functional reserve (Fig. 4, 6, 8). Given the relatively high response rate to lenvatinib in patients with poorly differentiated HCC [45] and the effect of lenvatinib on massive, confluent multinodular, and single nodular with extranodular growth tumor types, LEN-TACE sequential therapy is a rational and effective treatment strategy for patients who do not benefit from TACE alone and are susceptible to deterioration of hepatic functional reserve (e.g., ALBI grade 2).

\section{Conclusion}

This editorial described three HCC conditions that are unsuitable for TACE: conditions that easily become refractory to TACE, conditions in which hepatic functional reserve tends to deteriorate to Child-Pugh class B, and tumor pathology indicative of TACE resistance (Table 4). These types of intermediate-stage HCC should receive initial treatment with lenvatinib without TACE followed by lenvatinib pretreatment and selective TACE, which will maximize the efficacy of TACE and improve overall survival over that of patients receiving the current standard of care, namely, TACE alone [34]. Because lenvatinib therapy and TACE are complementary rather than competitive, they are both essential and should be used synergistically.

Further advances in the development of new anticancer agents may lead to a paradigm shift in the treatment of HCC, and systemic therapy will become the first choice of treatment followed by curative/selective TACE for the treatment of intermediate-stage HCC with high tumor burden (Fig. 7, 8).

The concept of TACE refractoriness was initially proposed in Japan and later spread worldwide. However, this concept is becoming outdated as a more important concept - TACE unsuitability - is being proposed and established in Japan and hopefully disseminated to the world.

\section{Disclosure Statement}

Masatoshi Kudo received lecture fees from Bayer, Eisai, MSD, and Ajinomoto, research grants from Chugai, Otsuka, Takeda, Taiho, Sumitomo Dainippon, Daiichi Sankyo, MSD, Eisai, Bayer, AbbVie, Medico's Hirata, Astellas Pharma, and Bristol-Myers Squibb, and advisory consulting fees from Kowa, MSD, BristolMyers Squibb, Bayer, Chugai, Taiho, Eisai, and Ono Pharmaceutical.

\section{References}

1 Cheng AL, Kang YK, Chen Z, Tsao CJ, Qin S, Kim JS, et al. Efficacy and safety of sorafenib in patients in the AsiaPacific region with advanced hepatocellular carcinoma: a phase III randomised, double-blind, placebocontrolled trial. Lancet Oncol. 2009 Jan;10(1):25-34.

2 Llovet JM, Ricci S, Mazzaferro V, Hilgard P, Gane E, Blanc JF, et al.; SHARP Investigators Study Group. Sorafenib in advanced hepatocellular carcinoma. N Engl J Med. 2008 Jul;359(4):378-90.

3 Kudo M, Finn RS, Qin S, Han KH, Ikeda K, Piscaglia F, et al. Lenvatinib versus sorafenib in first-line treatment of patients with unresectable hepatocellular carcinoma: a randomised phase 3 non-inferiority trial. Lancet. 2018 Mar;391(10126):1163-73.

4 Bruix J, Qin S, Merle P, Granito A, Huang YH, Bodoky G, et al.; RESORCE Investigators. Regorafenib for patients with hepatocellular carcinoma who progressed on sorafenib treatment (RESORCE): a randomised, doubleblind, placebo-controlled, phase 3 trial. Lancet. 2017 Jan;389(10064):56-66.

5 Abou-Alfa GK, Meyer T, Cheng AL, El-Khoueiry AB, Rimassa L, Ryoo BY, et al. Cabozantinib in Patients with Advanced and Progressing Hepatocellular Carcinoma. N Engl J Med. 2018 Jul;379(1):54-63. 
6 Zhu AX, Kang YK, Yen CJ, Finn RS, Galle PR, Llovet JM, et al.; REACH-2 study investigators. Ramucirumab after sorafenib in patients with advanced hepatocellular carcinoma and increased $\alpha$-fetoprotein concentrations (REACH-2): a randomised, double-blind, placebo-controlled, phase 3 trial. Lancet Oncol. 2019 Feb;20(2):28296.

7 Kudo M. Heterogeneity and Subclassification of Barcelona Clinic Liver Cancer Stage B. Liver Cancer. 2016 Apr; 5(2):91-6.

8 Galle PR, Forner A, Llovet JM, Mazzaferro V, Piscaglia F, Raoul JL, et al.; European Association for the Study of the Liver. EASL Clinical Practice Guidelines: management of hepatocellular carcinoma. J Hepatol. 2018 Jul; 69(1):182-236.

9 Marrero JA, Kulik LM, Sirlin CB, Zhu AX, Finn RS, Abecassis MM, et al. Diagnosis, Staging, and Management of Hepatocellular Carcinoma: 2018 Practice Guidance by the American Association for the Study of Liver Diseases. Hepatology. 2018 Aug;68(2):723-50.

10 Omata M, Cheng AL, Kokudo N, Kudo M, Lee JM, Jia J, et al. Asia-Pacific clinical practice guidelines on the management of hepatocellular carcinoma: a 2017 update. Hepatol Int. 2017 Jul;11(4):317-70.

11 Korean Liver Cancer Association KL, National Cancer Center NC; Korean Liver Cancer Association; National Cancer Center. 2018 Korean Liver Cancer Association-National Cancer Center Korea Practice Guidelines for the Management of Hepatocellular Carcinoma. Gut Liver. 2019 May;13(3):227-99.

12 Bruix J, Reig M, Sherman M. Evidence-Based Diagnosis, Staging, and Treatment of Patients With Hepatocellular Carcinoma. Gastroenterology. 2016 Apr;150(4):835-53.

13 Bolondi L, Burroughs A, Dufour JF, Galle PR, Mazzaferro V, Piscaglia F, et al. Heterogeneity of patients with intermediate (BCLC B) Hepatocellular Carcinoma: proposal for a subclassification to facilitate treatment decisions. Semin Liver Dis. 2012 Nov;32(4):348-59.

14 Kudo M, Arizumi T, Ueshima K, Sakurai T, Kitano M, Nishida N. Subclassification of BCLC B Stage Hepatocellular Carcinoma and Treatment Strategies: Proposal of Modified Bolondi's Subclassification (Kinki Criteria). Dig Dis. 2015 Oct;33(6):751-8.

15 Kudo M. Extremely High Objective Response Rate of Lenvatinib: Its Clinical Relevance and Changing the Treatment Paradigm in Hepatocellular Carcinoma. Liver Cancer. 2018 Sep;7(3):215-24.

16 Yamakado K, Hirota S. Sub-classification of intermediate-stage (Barcelona Clinic Liver Cancer stage-B) hepatocellular carcinomas. World J Gastroenterol. 2015 Oct;21(37):10604-8.

17 Mazzaferro V, Llovet JM, Miceli R, Bhoori S, Schiavo M, Mariani L, et al.; Metroticket Investigator Study Group. Predicting survival after liver transplantation in patients with hepatocellular carcinoma beyond the Milan criteria: a retrospective, exploratory analysis. Lancet Oncol. 2009 Jan;10(1):35-43.

18 Kudo M, Matsui O, Izumi N, Iijima H, Kadoya M, Imai Y, et al.; Liver Cancer Study Group of Japan. JSH ConsensusBased Clinical Practice Guidelines for the Management of Hepatocellular Carcinoma: 2014 Update by the Liver Cancer Study Group of Japan. Liver Cancer. 2014 Oct;3(3-4):458-68.

19 Kudo M, Matsui O, Izumi N, Kadoya M, Okusaka T, Miyayama S, et al.; Liver Cancer Study Group of Japan. Transarterial chemoembolization failure/refractoriness: JSH-LCSGJ criteria 2014 update. Oncology. 2014;87 Suppl $1: 22-31$

20 Raoul JL, Gilabert M, Piana G. How to define transarterial chemoembolization failure or refractoriness: a European perspective. Liver Cancer. 2014 May;3(2):119-24.

21 Management consensus guideline for hepatocellular carcinoma: 2016 updated by the Taiwan Liver Cancer Association and the Gastroenterological Society of Taiwan. J Formos Med Assoc. 2018 May;117(5):381-403.

22 Cheng AL, Amarapurkar D, Chao Y, Chen PJ, Geschwind JF, Goh KL, et al. Re-evaluating transarterial chemoembolization for the treatment of hepatocellular carcinoma: consensus recommendations and review by an international expert panel. Liver Int. 2014 Feb;34(2):174-83.

23 Ogasawara S, Chiba T, Ooka Y, Kanogawa N, Motoyama T, Suzuki E, et al. Efficacy of sorafenib in intermediatestage hepatocellular carcinoma patients refractory to transarterial chemoembolization. Oncology. 2014; $87(6): 330-41$.

24 Arizumi T, Ueshima K, Minami T, Kono M, Chishina H, Takita M, et al. Effectiveness of Sorafenib in patients with transcatheter arterial chemoembolization (TACE) refractory and intermediate-stage hepatocellular carcinoma. Liver Cancer. 2015 Dec;4(4):253-62.

25 Peck-Radosavljevic M, Kudo M, Raoul J, et al: Outcomes of patients (pts) with hepatocellular carcinoma (HCC) treated with transarterial chemoembolization (TACE): Global OPTIMIS final analysis. J Clin Oncol. 2018 May; 36(15 suppl):4018.

26 Arizumi T, Minami T, Chishina H, Kono M, Takita M, Yada N, et al. Time to Transcatheter Arterial Chemoembolization Refractoriness in Patients with Hepatocellular Carcinoma in Kinki Criteria Stages B1 and B2. Dig Dis. 2017;35(6):589-97.

27 Yasui Y, Tsuchiya K, Kurosaki M, Takeguchi T, Takeguchi Y, Okada M, et al. Up-to-seven criteria as a useful predictor for tumor downstaging to within Milan criteria and Child-Pugh grade deterioration after initial conventional transarterial chemoembolization. Hepatol Res. 2018 May;48:442-50.

28 Kimura H, Ohkawa K, Miyazaki M, Sakakibara M, Imanaka K, Tamura T, et al. Subclassification of patients with intermediate-stage (Barcelona Clinic Liver Cancer stage-B) hepatocellular carcinoma using the up-to-seven criteria and serum tumor markers. Hepatol Int. 2017 Jan;11(1):105-14. 
Kudo: New Treatment Strategy for TACE-Unsuitable Intermediate-Stage HCC

29 Eso Y, Takai A, Takahashi K, Ueda Y, Taura K, Marusawa H, et al. Combination of Mac-2 Binding Protein Glycosylation Isomer and Up-To-Seven Criteria as a Useful Predictor for Child-Pugh Grade Deterioration after Transarterial Chemoembolization for Hepatocellular Carcinoma. Cancers (Basel). 2019 Mar;11(3):11.

30 Izumoto H, Hiraoka A, Ishimaru Y, Murakami T, Kitahata S, Ueki H, et al. Validation of Newly Proposed Time to Transarterial Chemoembolization Progression in Intermediate-Stage Hepatocellular Carcinoma Cases. Oncology. 2017;93 Suppl 1:120-6.

31 Hiraoka A, Kumada T, Kudo M, Hirooka M, Koizumi Y, Hiasa Y, et al.; Real-life Practice Experts for HCC (RELPEC) Study Group and HCC 48 Group (hepatocellular carcinoma experts from 48 clinics). Hepatic Function during Repeated TACE Procedures and Prognosis after Introducing Sorafenib in Patients with Unresectable Hepatocellular Carcinoma: multicenter Analysis. Dig Dis. 2017;35(6):602-10.

32 Kanai T, Hirohashi S, Upton MP, Noguchi M, Kishi K, Makuuchi M, et al. Pathology of small hepatocellular carcinoma. A proposal for a new gross classification. Cancer. 1987 Aug;60(4):810-9.

33 Yamashita Y, Matsukawa T, Arakawa A, Hatanaka Y, Urata J, Takahashi M. US-guided liver biopsy: predicting the effect of interventional treatment of hepatocellular carcinoma. Radiology. 1995 Sep;196(3):799-804.

34 Kudo M, Ueshima K, Chan S, Minami T, Chishina H, Aoki T, et al. Lenvatinib as an Initial Treatment in Patients with Intermediate-Stage Hepatocellular Carcinoma Beyond Up-To-Seven Criteria and Child-Pugh A Liver Function: A Proof-Of-Concept Study. Cancers (Basel). 2019 Jul;11(8):11.

35 Yamashita T, Kudo M, Ikeda K, et al. REFLECT-A phase 3 trial comparing efficacy and safety of lenvatinib to sorafenib for the treatment of unresectable hepatocellular carcinoma: a subset analysis of Japanese patients. J Gastroenterol. Forthcoming 2019.

36 Ueshima K, Nishida N, Hagiwara S, Aoki T, Minami T, Chishina H, et al. Impact of Baseline ALBI Grade on the Outcomes of Hepatocellular Carcinoma Patients Treated with Lenvatinib: A Multicenter Study. Cancers (Basel). 2019 Jul;11(7):11.

37 Kano MR, Komuta Y, Iwata C, Oka M, Shirai YT, Morishita Y, et al. Comparison of the effects of the kinase inhibitors imatinib, sorafenib, and transforming growth factor-beta receptor inhibitor on extravasation of nanoparticles from neovasculature. Cancer Sci. 2009 Jan;100(1):173-80.

38 Jain RK. Normalization of tumor vasculature: an emerging concept in antiangiogenic therapy. Science. 2005 Jan;307(5706):58-62.

39 Tohyama O, Matsui J, Kodama K, Hata-Sugi N, Kimura T, Okamoto K, et al. Antitumor activity of lenvatinib (e7080): an angiogenesis inhibitor that targets multiple receptor tyrosine kinases in preclinical human thyroid cancer models. J Thyroid Res. 2014;2014:638747.

40 Carmeliet P, Jain RK. Angiogenesis in cancer and other diseases. Nature. 2000 Sep;407(6801):249-57.

41 Kojiro M, Sugihara S, Kakizoe S, Nakashima O, Kiyomatsu K. Hepatocellular carcinoma with sarcomatous change: a special reference to the relationship with anticancer therapy. Cancer Chemother Pharmacol. 1989; 23(S1 Suppl):S4-8.

42 Zen C, Zen Y, Mitry RR, Corbeil D, Karbanová J, O'Grady J, et al. Mixed phenotype hepatocellular carcinoma after transarterial chemoembolization and liver transplantation. Liver Transpl. 2011 Aug;17(8):943-54.

43 Wang B, Xu H, Gao ZQ, Ning HF, Sun YQ, Cao GW. Increased expression of vascular endothelial growth factor in hepatocellular carcinoma after transcatheter arterial chemoembolization. Acta Radiol. 2008 Jun;49(5): 523-9.

44 Miyayama S. Ultraselective conventional transarterial chemoembolization: when and how? Clin Mol Hepatol. 2019 Apr. https://doi.org/10.3350/cmh.2019.0016.

45 Kudo M. Association between tumor response by mRECIST and overall survival in patients with poorly differentiated HCC in REFLECT study. APPLE; 2019. Available from: www.c-linkage.co.jp > apple2019 > doc > apple2019_program.pdf. 